\title{
Repeated administration of pyrithiamine leads to a proportional increase in the remembered durations of events
}

\author{
WARREN H. MECK \\ Columbia University, New York, New York \\ and \\ KATHRYN E. ANGELL \\ Brown University, Providence, Rhode Island
}

\begin{abstract}
Twenty rats were trained on a peak-interval (PI) timing procedure with auditory signals. For 10 rats, the time of reinforcement was set at $20 \mathrm{sec}$; for the remaining rats, the time of reinforcement was set at $50 \mathrm{sec}$. After seven sessions of baseline training, $4 \mathrm{mg} / \mathrm{kg}$ of pyrithiamine (PT) were administered i.p. to half of the rats in each group before each of six test sessions, and physiological saline was administered to the remaining subjects. During testing, the times of the maximum response rates of PI functions for PT-treated rats gradually shifted rightward on the time scale until they occurred approximately $14 \%$ later than the times of the maximum response rates of the rats receiving saline injections. This pattern of timing behavior can be interpreted as reflecting an increase in the remembered times of reinforcement associated with a decrease both in the effective levels of acetylcholine and in the speed of memory storage.
\end{abstract}

Pyrithiamine (PT) is a major antimetabolite of thiamine. As a thiamine antagonist, short-term PT administration has been used as a model of thiamine deficiency that can lead to a relatively specific inhibition of acetylcholine (ACh) synthesis by limiting pyruvate dehydrogenase activity (see, e.g., Cheney, Gubler, \& Jaussi, 1969; Cooper \& Pincus, 1967; Eder, Hirt, \& Dunant, 1976; Mann \& Quastel, 1940; Vorhees, Schmidt, Barrett, \& Schenker, 1977). The transformation of pyruvate to acetyl-coenzyme $A$, which is combined with choline to form $\mathrm{ACh}$, is made with the support of pyruvate dehydrogenase, a thiamine-dependent catalyst. Since PT acts centrally to decrease thiamine levels, pyruvate dehydrogenase activity is decreased, lowering the amount of acetyl-coenzyme $A$ that is produced. Diminished acetyl-coenzyme $A$ levels lead to diminished ACh levels, and as a result, we propose that memory storage speed, which is dependent on the effective level of ACh (see Meck \& Church, 1987a), is decreased and thereby induces a proportional increase in the remembered times of reinforcement. This change in timing behavior is reflected by the maintenance of a rightward horizontal

\footnotetext{
The preparation of this manuscript was supported in part by a research grant from the National Institute of Neurological and Communicative Disorders and Stroke: NS 24794 and by an Alfred P. Sloan Foundation Research Fellowship awanded to W.H.M. at Columbia University. The authors would like to acknowledge Russell M. Church for the generous use of his laboratory and for his advice and comments on earlier versions of this article. A portion of this work was presented at the 56th Annual Meeting of the Eastern Psychological Association in Boston (Angell \& Meck, 1985). Requests for reprints should be addressed to Warren H. Meck, Department of Psychology, 406 Schermerhorn Hall, Columbia University, New York, NY 10027.
}

displacement of interval timing functions. Furthermore, because of the relevance of PT treatment to various disease states affecting human memory processes, such as Wernicke-Korsakoff syndrome (e.g., McEntee, Mair, \& Langlais, 1984; Troncoso, Johnson, Hess, Griffin, \& Price, 1981) and carbohydrate-utilization disorders (e.g., Parker et al., 1984), we were interested in evaluating its effect on temporal memory. In this regard, however, it should be noted from the outset that much of the research relating thiamine deficiency to animal models of Korsakoff's psychosis has focused on the depletion of cortical norepinephrine and thalamic lesions induced by the combined effects of daily administration of PT and maintenance on a thiamine-deficient diet, thereby implicating neurotransmitter systems other than ACh in this type of mental disorder (e.g., Mair, Anderson, Langlais, \& McEntee, 1985, 1988; Mair \& McEntee, 1983).

Hashimoto (1981) reported that PT administration altered timing behavior in rats trained to hold down a lever for a fixed duration in order to receive a food reward. In this differential reinforcement of low-rate (DRL) schedule, no external stimulus was used to signal the end of the required duration, and the minimum temporal criterion was gradually increased until it was maintained at $2 \mathrm{sec}$. Daily injections of PT were given after the daily 10-min experimental sessions. The continuous administration of PT at $1 \mathrm{mg} / \mathrm{kg}$ (i.p.) produced no change in the response-duration functions during the entire course of the experiment. For subjects administered $2 \mathrm{mg} / \mathrm{kg}$, the initial response durations $(2.06 \pm 0.03)$ became significantly longer $(+6 \%, p<.02)$ after Day 8 . However, this incremental tendency was rather weak, and on Day 14 (the 
last day), the overall mean of the response-duration function was $2.22 \pm 0.03 \sec (+9 \%, p<.01)$. After the injections were discontinued, the prolongation of the response duration gradually diminished, and, within a month, it recovered to the control level. This prolongation was still present after administration of thiamine $(1 \mathrm{mg} / \mathrm{kg})$ for 7 days, but a large dose of thiamine $(20 \mathrm{mg} / \mathrm{kg})$ produced a rapid recovery. Under the last condition, the full recovery was achieved in 2-4 days. Finally, in the 4-mg/kg-PT group, the mean of the response-duration function was significantly increased only 2 days after the first administration $(+4 \%, p<.01)$. This tendency was extended until Day $9(+18 \%)$, and its overall mean for the response duration was maintained at $2.43 \pm 0.03 \mathrm{sec}$. The distribution of reinforced responses shifted rightward, and the peak of the distribution was not as sharply defined as that of the controls.

The ability of animals to measure signal durations in the seconds-to-minutes range can be evaluated by a number of different behavioral procedures, each with its own virtues (Church, 1989; Gibbon \& Church, 1990). One of the procedures that has been used extensively in order to evaluate the content of temporal memory is the peakinterval (PI) timing procedure (Church, 1984; Gibbon, Church, \& Meck, 1984). The PI procedure is an extension of the well-known fixed-interval (FI) procedure (e.g., Dews, 1970) and is designed to provide independent measures of several behavioral processes involved in temporal discriminations. In a signaled, discrete-trial version of the PI procedure, some trials are identical to those of a FI procedure; other trials consist of a signal that continues well beyond the fixed duration, and no reinforcement is available. On the nonreinforced or peak trials, the animal's response rate increases as a function of time, until a point near the time that reinforcement is sometimes made available, and then it decreases in a fairly symmetrical manner (e.g., Church, Miller, Meck, \& Gibbon, 1991). The time during which the response rate is maximal is called the peak time. It occurs near the time that reinforcement is maximally expected by the subject. The response rate at the peak time is called the peak rate. Various experimental manipulations have shown that peak time (horizontal placement) and peak rate (vertical placement) are independent measures of timing performance because either of the measures can be changed without a concomitant change in the other measure (e.g., Meck, 1987; Roberts, 1981).

The separation of clock and memory functions is currently one of the most challenging problems in the field of timing and time perception. Changes in clock speed produce phasic horizontal shifts in time-discrimination functions; changes in the content of temporal memory produce chronic horizontal shifts in time-discrimination functions. The phasic changes characteristic of changes in clock speed have been shown to be related to changes in dopaminergic function; the chronic changes characteris- tic of changes in memory storage speed have been shown to be related to changes in cholinergic function (e.g., Church \& Meck, 1988; Meck, 1983; Meck \& Church, 1987a, 1987b). Recent evidence indicates that the content of temporal memory can be selectively modified in a proportional manner by certain drugs, nutrients, brain lesions, and aging processes (e.g., Meck, 1983, in press; Meck \& Church, 1987a, 1987b; Meck, Church, \& Olton, 1984; Meck, Church, \& Wenk, 1986, in press; Meck, Church, Wenk, \& Olton, 1987). The neuropharmacological mechanisms common to each of these treatments appear to be dependent to a large extent on the neural activity controlled by the release of ACh. The purpose of the present experiment was to extend these findings by investigating the effects of PT on temporal memory in rats with the use of PI 20-sec and PI 50-sec timing procedures.

\section{METHOD}

\section{Subjects}

Twenty experimentally naive male Sprague-Dawley rats (Charles River CD). The rats weighed an average of $400 \mathrm{~g}$ each and were approximately 300 days old when the experiment began. They were individually housed in suspended metal cages (Wahmann Mfg. Co., Baltimore, MD). Water was provided continuously in the home cage, and rats were fed $11 \mathrm{~g}$ of ground rat chow mixed with $11 \mathrm{ml}$ of water each day within $15 \mathrm{~min}$ of the completion of the daily experimental sessions. A 12:12-h light:dark cycle was in effect in the colony room for the duration of the experiment, with fluorescent lights on from 6 a.m. to 6 p.m. Eastern Standard Time. All experimental sessions were conducted within 20 min of the same time each day during the light phase of the light:dark cycle.

\section{Apparatus}

Experimental sessions took place in 10 similar lever boxes that measured $23 \times 20 \times 22 \mathrm{~cm}$. The roof and side walls were transparent acrylic, whereas the narrower front and back walls were aluminum. The floor was constructed of 16 parallel stainless steel bars. A pellet dispenser (Gerbrands Model D-1 or Davis Scientific Instruments Model PD-104) delivered 45-mg Noyes Precision food pellets to a food cup through an opening in the front wall. A 140-ml glass water bottle, at least half full, hung from the back wall of the lever box. Each box contained a stainless steel lever to the left of the food cup. The levers in Boxes $1-6$ were $1.2 \times 3.4 \mathrm{~cm}$ and were located $3.6 \mathrm{~cm}$ above the grid floor (Coulbourn Instruments Model E223-05). The levers in Boxes 7-10 were $2.5 \times 5.0 \mathrm{~cm}$ and were located $5.0 \mathrm{~cm}$ above the floor (BRS/LVE Model 123-07). Each lever box was housed in a large insulation-board chamber designed to minimize outside light or sound. Boxes 1-6 had a 7.5-W, $100-\mathrm{V}$ lamp attached to the middle of the back wall of the chamber; Boxes 7-10 had a 6-W, 100-V lamp attached to the outside of the roof of the lever box. A Grason-Stadler noise generator (Model $901 \mathrm{~B}$ ) could deliver white noise of about $80 \mathrm{~dB}$ (re $20 \mu \mathrm{N} / \mathrm{m}^{2}$; General Radio Sound Lever Meter, Model 1565-D, A scale) above background level through a 4-in. $(10.2-\mathrm{cm})$ speaker mounted inside each chamber. Each chamber was equipped with a fan for ventilation and a small acrylic window for observation. A time-shared Digital Equipment Corporation PDP-12 computer controlled the experimental equipment and recorded the data. The sampling rate was $10 / \mathrm{sec}$, so that the smallest unit of time in which responses could be recorded was $0.1 \mathrm{sec}$, and the maximum observable response rate was 600 responses per minute. 


\section{Drug Administration}

PT $(4 \mathrm{mg} / \mathrm{kg}$ ) dissolved in $0.2 \mathrm{cc}$ of physiological saline $(0.9 \%$ $\mathrm{NaCl}$ ) or an equal volume of saline alone was administered to each rat intraperitoneally (i.p.) 20 min before the start of each day's experimental session.

\section{Experimental Design}

Twenty rats were randomly divided into two groups. For the first group, food, when available, was primed at $20 \mathrm{sec}$; for the second group, food, when available, was primed at $50 \mathrm{sec}$. These two groups were each divided into two squads: saline injections were administered before experimental training sessions for the first squad and PT injections were administered before experimental training sessions for the second squad. A group of 10 rats with a common time of reinforcement was trained on alternating days. Experimental sessions lasted for $2.5 \mathrm{~h}$. After the first drug-training session, on days when the animals did not receive PI training, each animal received the opposite type of injection. Thus, over the entire experiment, each subject was administered equal numbers of both saline and PT injections on alternating days. The critical difference was that half of the subjects received PI training only following saline injections and the remaining animals received PI training only following PT injections. The injections were identical in dosage, vehicle, and volume to those described above.

\section{Behavioral Procedures}

Pretraining. Each rat received at least two sessions of magazine and lever training. During these sessions, a pellet of food was delivered once every $30 \mathrm{sec}$, and, in addition, each leverpress produced food. The session began with the insertion of the response lever and continued until either the rat had pressed the lever 60 times or $30 \mathrm{~min}$ had passed, whichever came first. The houselight illuminated the lever box during the training session.

Peak-interval baseline training (Sessions 1-7). All rats were trained on a discrete-trials PI timing procedure, with one group of rats receiving 20-sec PI training and the other group receiving $50-\mathrm{sec}$ PI training. The onset of a white-noise signal marked the beginning of each trial. A random half of the trials were food trials. On these trials, food was made available either $20 \mathrm{sec}$ or $50 \mathrm{sec}$ after the onset of a white-noise signal, and the rat's first leverpress after the fixed duration terminated the noise signal and activated the pellet dispenser. The remaining half of the trials were peak trials. On these peak trials, food was not made available at $20 \mathrm{sec}$ or $50 \mathrm{sec}$, and the noise signal continued uninterrupted for a total of $130 \mathrm{sec}$. All trials were separated by a $130-\mathrm{sec}$ fixed-duration intertrial interval. In the PI procedure, data are collected exclusively from the peak trials for which peak-time and peak-rate measures are calculated.

Peak-interval drug testing (Sesdions 8-13). These sessions were identical to Sessions 1-7, except that squads of rats were injected either with saline or PT 20 min prior to the start of the experimental session. Data continued to be collected only during the peak trials.

Peak-interval postdrug testing (Session 14). This session was identical to Sessions 8-13, except that all squads of rats were injected with saline 20 min prior to the start of the experimental session. Data continued to be collected only during the peak trials.

\section{Data Analysis}

Only responses made during peak trials were recorded for later analysis. Responses were collected in 1-sec bins that corresponded to the number of seconds elapsed since signal onset. Response distributions were tallied for each day's performance for each rat. Peak times were determined by a method described by Meck, Church, and Olton (1984). Median peak times for Days 3-7 and for Days 9-13 were calculated for each rat under both control and drug conditions. These values were used to determine ratios of experimental peak times to control peak times for each subject, thus giving a signed percentage change in peak time as a function of drug treat- ment. The resulting values were used in a two-factor analysis of variance (ANOVA) in order to analyze whether time of reinforcement and/or drug condition had an effect on peak time. Maximum response rates were also evaluated. ANOVAs were performed on the response rates at the peak times for both baseline and experimental values.

\section{RESULTS}

PT administration resulted in the experience-dependent development of a rightward shift in both the 20-sec and the 50-sec PI timing functions. Peak-time measures for each of the squads (control and experimental) in the 20-sec PI group (means \pm standard errors) are plotted as a function of Sessions 3-14 in Figure 1. Similar measures for each of the squads in the 50-sec PI group are plotted in Figure 2. The final test session (Session 14), during which all subjects received saline injections prior to testing, is also plotted in these figures. Taken together, these figures illustrate the incremental nature of the rightward shift in peak time induced by PT administration. Systematic trends in the peak-time measures were evaluated using two-factor repeated measures ANOVAs. During baseline training (Sessions 3-7), there were no significant effects of condition (control vs. experimental), sessions (repeated measure), or a condition $\times$ sessions interaction for either the 20-sec group $[F(1,8)=0.002, F(4,32)=0.57$, and $F(4,32)=0.92$, respectively, all n.s. $(p>.05)]$ or the $50-\sec$ group $[F(1,8)=0.13, F(4,32)=1.29$, and $F(4,32)=1.18$, respectively, all n.s. $(p>.05)]$. In contrast, during drug testing (Sessions 8-13), although there were nonsignificant effects of condition (control vs. experimental), there were significant effects of sessions (repeated measure) and a significant condition $\times$ sessions interaction for the 20-sec group $[F(1,8)=3.46, p>.05$, $F(5,40)=3.43, p<.05$, and $F(5,40)=2.75, p<.05$, respectively]. Likewise, for the 50 -sec group during drug testing (Sessions 8-13), there were significant effects of condition (control vs. experimental), nonsignificant effects of sessions (repeated measure), and a significant condition $\times$ session interaction $[F(1,8)=14.0, p<.01$, $F(5,40)=2.0, p>.05$, and $F(5,40)=2.6, p<.05$, respectively]. In no case was any significant difference in peak time observed between Session 13 and Session 14 for any of the squads $[t s(4)<1, n . s$.].

Median response rate functions normalized by each subject's maximum response rate for each of the squads (control and experimental) in the 20-sec PI group are plotted for both training and testing phases in Figure 3. Similar measures for each of the squads in the 50-sec PI group are plotted in Figure 4. Taken together, these graphs illustrate the form and horizontal placement of the peak functions during each phase of the experiment. The percentage of maximum response rate is used to equalize the contribution of each rat's response function in determining the horizontal placement of the average peak function on the time scale.

Means and standard errors were calculated for the ratios of the individual median testing-session peak times 


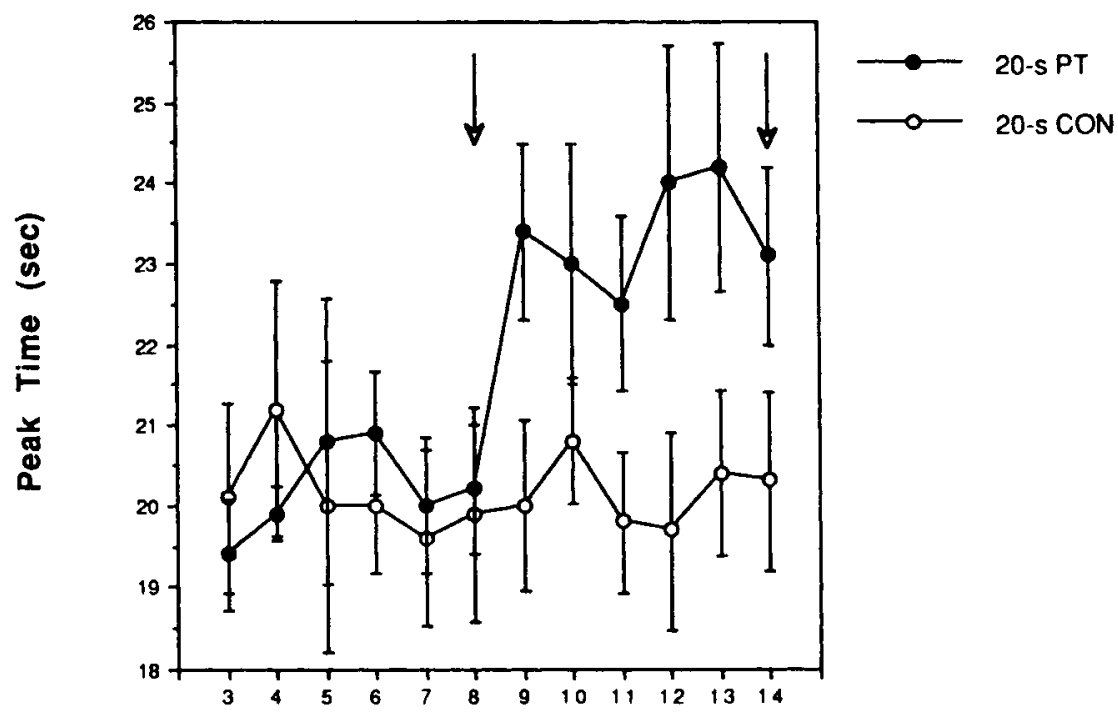

\section{Sessions}

Figure 1. Mean peak time \pm the standard error as a function of training (Sessions 3-7) and testing (Sessions 8-14) for subjects performing on a 20-sec PI timing procedure. Arrows indicate the occurrence of the first of six test sessions following saline control (CON) or pyrithiamine (PT) injections (Sescion 8) and the single drug-free test sescion following the drug test phase (Sescion 14).

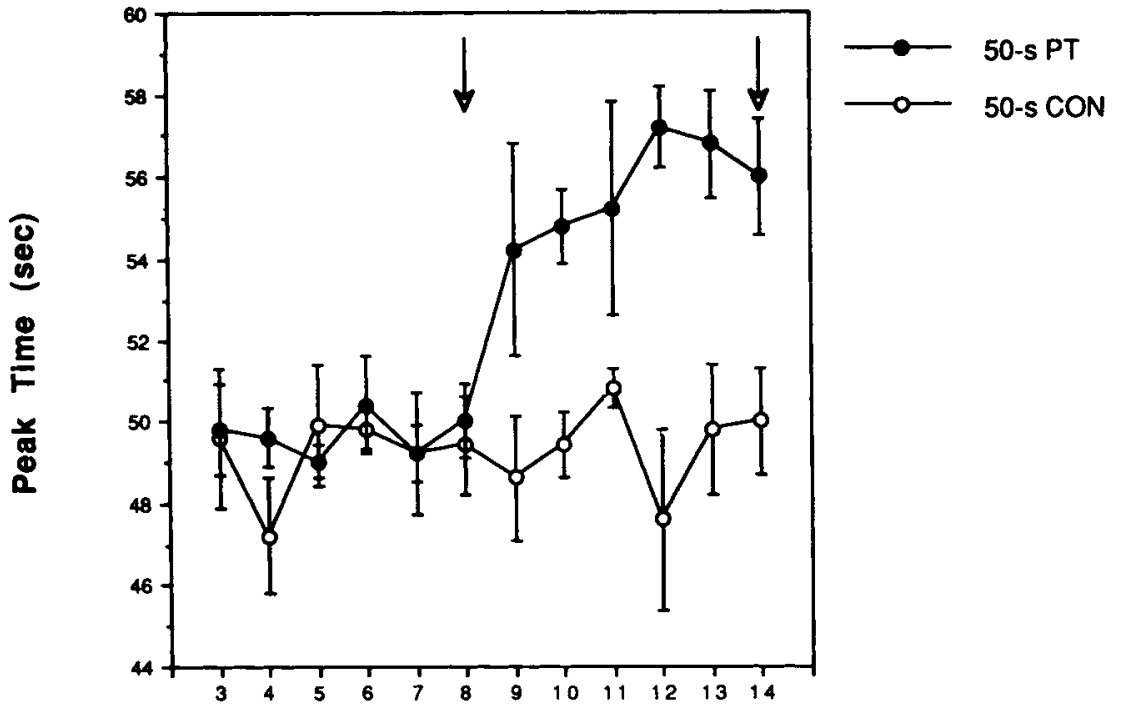

Sessions

Figure 2. Mean peak time \pm the standard error as a function of training (Sessions 3-7) and testing (Sesdons 8-14) for subjects performing on a 50-sec PI timing procedure. Arrows indicate the occurrence of the first of six test sesdions following saline control (CON) or pyrthiamine (PT) injections (Sesion 8) and the singie drug-tree test secion following the drug test phese (Sevion 14). 

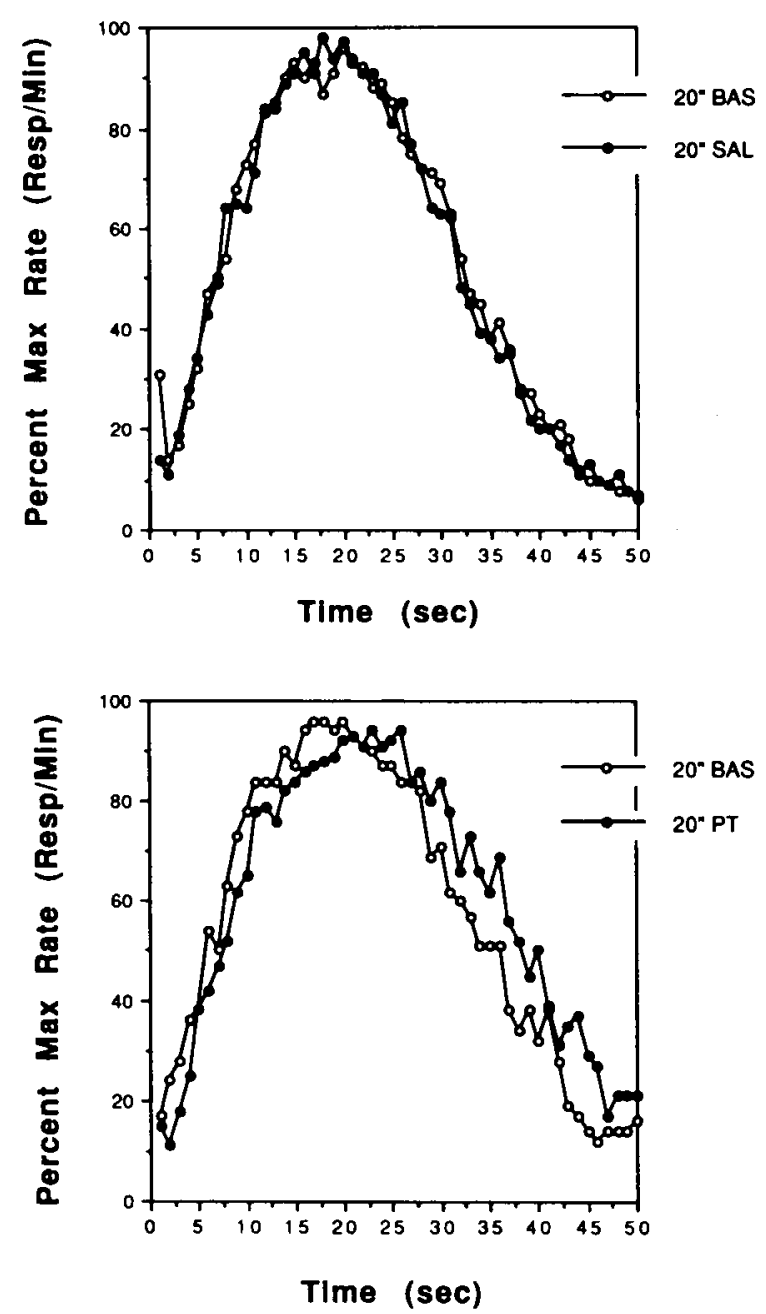

Figure 3. Median percent maximum response rate functions for the group of rats performing on the 20-sec PI timing procedure and divided into squads of rats recelving the control treatment sequence (upper panel)-that b, beseline training (BAS) followed by solline (SAL) injection testing-or the experimental treatment sequence (ower panel), which consisted of bockine training (BAS) followed by pyrithiamine (PT) injection testing.

divided by the individual median training-session peak times for each squad of rats during the last three sessions of each phase (Sessions 5-7 and Sessions 11-13). For the $20-\mathrm{sec}$ group, the mean ratio for the squad of rats tested under the influence of saline was $0.99 \pm 0.02$; for the squad of rats tested under the influence of PT, it was $1.14 \pm 0.04$. For the 50 -sec group, the ratio for rats tested under the influence of saline was $1.00 \pm 0.01$; for the squad of rats tested under the influence of PT, it was $1.14 \pm 0.01$. A two-factor ANOVA for the percentage change measure showed this $14 \%$ increase in peak time as a function of PT administration (control vs. experimental) to be significant $[F(1,16)=38.98, p<.001]$, whereas the effects of time of reinforcement $(20 \mathrm{sec}$ vs. $50 \mathrm{sec})$ and the interaction between condition and time of rein- forcement were found to be unreliable $\left[F_{\mathrm{s}}(1,16)=0.19\right.$ and 0.07 , respectively, all n.s.].

Means and standard errors were calculated for the individual median peak response rates for the four squads of rats. A comparison of these peak rates was made between the last three baseline training sessions (Sessions 5-7) and the last three drug testing sessions (Sessions 11-13). For the 20-sec group, the mean peak rate for the squad of rats initially trained without injections and later tested under the influence of saline injections was $69 \pm 7.82$ and $68.8 \pm 4.79$, respectively; for the squad of rats initially trained without injections and later tested under the influence of PT injections, it was $57.2 \pm 11.26$ and $56.0 \pm 7.09$, respectively. For the 50 -sec group, the mean peak rate for the squad of rats initially trained without injections
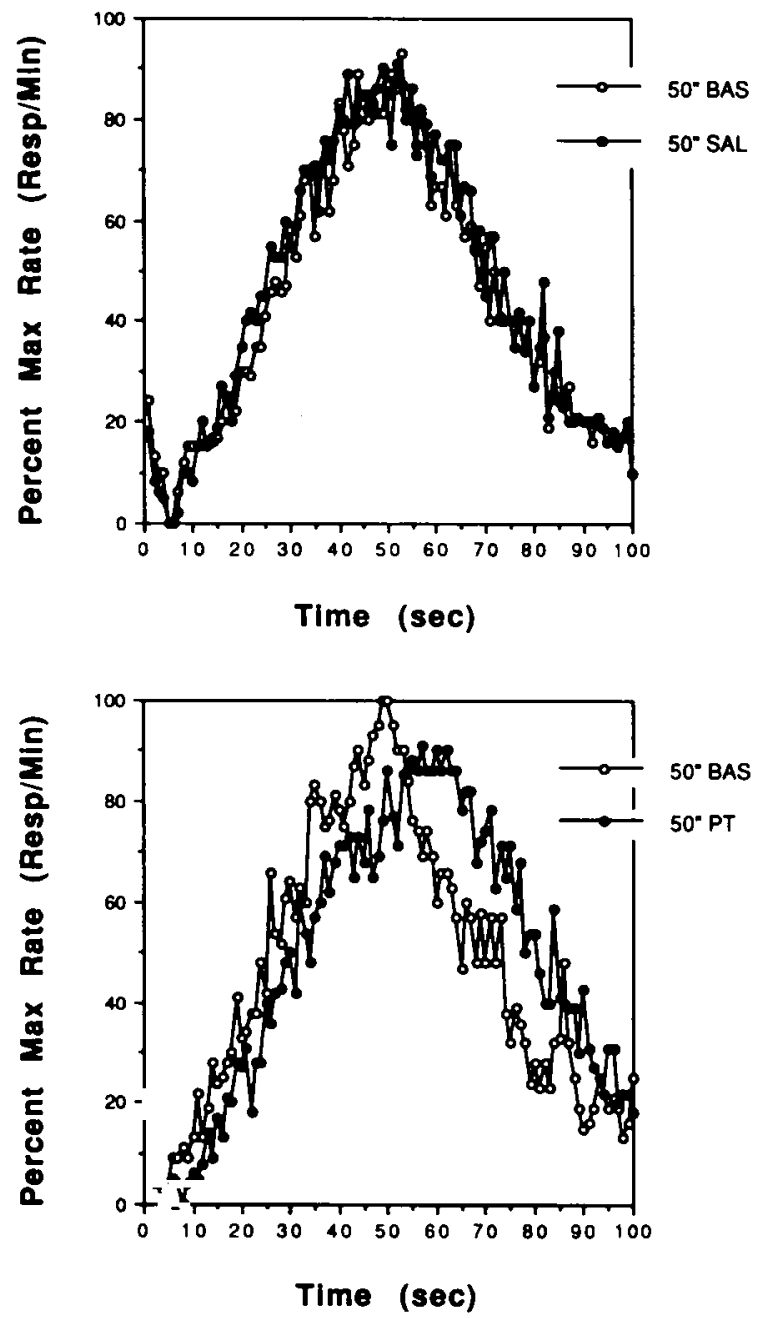

Figure 4. Median percent maximum response rate functions for the group of rats performing on the SO-wec PI timing procedure and divided tite squads of rats recelving the control trentment sequesce (upper panei)-that b, beseline training (BAS) followed by saline (SAL) injection teating-or the experimental trentment sequence (lower panel), which consteded of baseline training (BAS) followed by pyrithiamine (PT) injection testing. 
and later tested under the influence of saline injections was $60.6 \pm 8.95$ and $60.6 \pm 7.57$, respectively; for the squad of rats initially trained without injections and later tested under the influence of PT injections it was $53.0 \pm 5.44$ and $53.4 \pm 4.39$, respectively. A three-factor repeated measures ANOVA indicated that the peak rate of responding was not influenced in a reliable fashion by differences in condition (control vs. experimental), time of reinforcement ( 20 vs. $50 \mathrm{sec}$ ), the interaction between condition and time of reinforcement, or the repeated measure factor [training vs. testing; $F \mathrm{~s}(1,16)=1.86,0.66,0.12$, and 0.02 , respectively, all n.s.].

\section{DISCUSSION}

The data presented here support the earlier findings reported by Hashimoto (1981) in demonstrating that timing behavior is sensitive to PT administration in a relatively specific manner. Hashimoto's discovery that PT administration produced a gradual rightward shift in the response functions for rats trained on a 2 -sec DRL schedule was extended in four major ways: (1) The horizontal displacement of the DRL timing function was generalized to a different task, the PI timing procedure, which generates a timing function that has a substantially different form and is indexed by different response measures than the DRL schedule, (2) the range of durations over which PT effects have been investigated was increased from the single value of $2 \mathrm{sec}$ to the much larger values of $20 \mathrm{sec}$ and $50 \mathrm{sec}$, (3) the magnitude of the rightward shift in response functions was shown to be proportional to the value of the signal being timed, and it was demonstrated that this effect is independent of any change in the responsiveness of the subject, and (4) the PT-induced behavioral effects observed in the experimental subjects were shown to be dependent on the proximity of the administration of PT to the behavioral test sessions. This conclusion is warranted because control subjects given an equal number of PT injections on alternate days (i.e., none on test days) did not exhibit any changes in timing behavior relative to the baseline training period, thus supporting an explanation involving memory consolidation.

As indicated above, an analysis of the peak functions for animals trained on a 20 -sec PI timing procedure and for animals trained on a 50-sec PI timing procedure indicated that PT administration increased the remembered durations of events in a proportional manner. Saline injections had no reliable effects on peak time in either procedure. Meck and Church (1987a, 1987b) described how an increase in peak time could result either from a decrease in clock speed or from a decrease in memory storage speed. Fortunately, these two potential explanations for the systematic misrepresentation of physical time result in two different patterns of timing behavior and hence can be distinguished. If a manipulation causes a decrease in clock speed, then the resulting timing func- tions will exhibit the following characteristics, which are collectively referred to as the clock pattern:

1. The peak time will occur later than normal during the first session in which the manipulation (i.e., drug or lesion) is applied, because the accumulator will be receiving pacemaker pulses less rapidly and therefore will not have collected the critical number of pulses required to meet the subject's temporal criterion until additional time has passed.

2. The peak time will gradually revert back to the baseline value over subsequent testing sessions as the manipulation continues to be applied. This recovery is the result of the animal learning that responses only at a value smaller than originally expected result in reinforcement; the new smaller time values will be transferred into reference memory, replacing the older larger values. As this acquisition process occurs, the remembered values will come to accurately reflect the time of reinforcement measured by the slowed clock, albeit on a different time scale than normal.

3. The difference between the original peak time and the peak time during the first sessions of the manipulation is proportional to the actual time of reinforcement. This is a direct result of the fact that the clock speed has been changed by a multiplicative constant.

4. When the manipulation is discontinued, the peak time abruptly jumps to a time less than the control peak time. Because the animal has already adapted to the new clock speed, as described above, then when it reverts to the former clock speed, it will once again go through the process described in the first characteristic, this time in the opposite direction with equal magnitude. If the experimentally obtained peak time for some other treatment is earlier than the control peak time, then the process described above will apply, only with the directions reversed (e.g., amphetamine increases clock speed and haloperidol decreases clock speed, see Meck, 1983).

A misrepresentation of physical time that originates during the transfer of a value from the accumulator to reference memory follows a different set of rules, which are collectively referred to as the memory pattern:

1. An increase in the memory constant $\left(\mathrm{K}^{*}\right)$, which may be interpreted as a decrease in the memory storage speed, initially results in no behavioral change when the treatment is applied. Over the first few sessions with the new manipulation, the peak of the response function gradually shifts to a larger time value. During the initial sessions, most of the sample time values in reference memory still accurately reflect the physical time of reinforcement as recorded by the internal clock. Consequently, during the first few sessions, the animal continues to respond at the appropriate time. However, since memory storage speed is being decreased, during each of these trials a later time value than normal is being stored in reference memory. As the rat experiences more trials, more of the sample time values in reference memory reflect a later time such 
that over the next few sessions, the animal will gradually come to start responding maximally at a later time than normal within the trial.

2. Since it is the actual memory of the time value recorded by the clock that is impaired, the animal cannot easily adjust its behavior to the alteration in memory storage processes. The values recorded in reference memory are consistently later than the actual times of reinforcement measured by the pacemaker system. Thus, the animal's peak time will continue to be late as long as the manipulation is applied and the distortion in memory storage speed continues.

3. Similar to the difference involved in the clock-speed effect, the difference between original peak time and experimental peak time in this situation will be proportional to the actual time of reinforcement. Because a change in memory storage speed also leads to a multiplicative constant, then the change in the remembered times of reinforcement will be proportional to the number of pacemaker pulses in the accumulator-that is, unless there has been a concomitant change in clock speed, the number of pulses should accurately reflect the time of reinforcement.

4. Finally, when the manipulation is removed, the peak time gradually reverts to the original peak time without ever moving to a time value significantly less than the time of reinforcement. Once the manipulation is discontinued, the incorrect time values stored in memory are gradually replaced by more accurate values such that the animal gradually learns to respond at the appropriate time once again. If a manipulation were used that effected an increase in memory storage speed instead of a decrease, then the similar changes would take place, except in the opposite direction.

The difference between baseline peak times and peak times under PT administration for both groups was approximately $14 \%$ of the actual time of reinforcement; the increase took the form of a constant percentage, not a constant number of seconds. This rightward shift of the peak function did not appear until Day 2 of PT administration. Once the animals had begun to respond maximally at a later time, this trend continued for the duration of PT administration and was maintained during the one test session without PT administration. These characteristics suggest that PT administration results in a memory effectthat is, the memory constant $\left(\mathrm{K}^{*}\right)$ was increased, reflecting a decrease in the speed of memory storage.

Previous studies have indicated that an alteration in memory storage speed is indicative of a change in brain ACh levels (e.g., Meck \& Church, 1987a, 1987b). There is evidence consistent with a role for $\mathrm{ACh}$ in the present experiment as well. PT mimics thiamine deficiency by inhibiting the conversion of thiamine to its active form, thiamine pyrophosphate. Thiamine pyrophosphate is a necessary coenzyme in pyruvate dehydrogenase, the enzyme that catalyzes the synthesis of acetyl-coenzyme A from pyruvate. ACh is then synthesized from acetylcoenzyme A, and choline by choline acetyl transferase. Cheney et al. (1969) demonstrated that brain ACh levels were decreased in both PT-treated and thiamine-deprived rats compared with thiamine-treated controls. In vivo injection of pyruvate reversed the decrease in ACh levels for PT-treated animals but not for thiamine-deprived animals. Parker et al. (1984) confirmed that brain pyruvate dehydrogenase activity was decreased in PT-treated animals that were also fed a low-thiamine diet. Evidence on whether or not thiamine-deficient diets result in decreased acetyl-coenzyme A levels (Heinrich, Stadler, \& Weiser, 1973; Reynolds \& Blass, 1975), decreased ACh levels (Heinrich et al., 1973; Reynolds \& Blass, 1975), or decreased pyruvate dehydrogenase activity (Gubler, 1961; Heinrich et al., 1973) remains inconsistent. Although the appropriate experimental work has not been conducted, it seems reasonable to suppose that $\mathrm{ACh}$ levels can be decreased by PT injection within the time periods used in the present study and that recovery in ACh levels would occur within a 24-h period for rats not deprived of thiamine and given PT injections every other day (Hashimoto, 1981; Rindi, Patrini, Comincioli, \& Reggiani, 1980; Rindi \& Perri, 1961; Sacchi et al., 1978).

In summary, it is clear that PT administration produced an effect that may be interpreted as an increase in the remembered times of reinforcement. The characteristics of the rightward shift of the peak functions are in accordance with the characteristics of the memory pattern: a gradual but permanent shift in peak time that reflects a difference in remembered times of reinforcement that is proportional to the actual times of reinforcement. Like atropine, PT exerts its effects, in part, by decreasing the effective levels of brain ACh. Since PT was administered to all rats, the only difference being whether they received PI testing following the PT injections or not, the observed effects on peak time are not due simply to repeated administration. Because the PI timing procedure discriminates between changes in peak time and changes in response rate, and the peak rates of animals receiving PT injections just prior to PI testing did not differ from the peak rates of animals given saline injections just prior to PI training, it is unlikely that the observed differences in timing behavior are due to either motor effects or changes in motivational levels. The proposed mechanism is, therefore, a decrease in the effective ACh levels through a decrease in pyruvate dehydrogenase activity, a decrease in memory storage speed, and a proportional increase in the remembered times of reinforcement:

\section{REFERENCES}

ANGELL, K. E., MECK, W. H. (1985, April). Repeated administra tion of pyrithiamine leads to a proportional increase in the remembered duration of events. Paper presented at the 56th Annual Meeting of the Eastern Psychological Association, Boston.

Cheney, D. L., Gubler, C. J., \& Jaussi, A. W. (1969). Production of acetylcholine in rat brain following thiamine deprivation and treatment with thiamine antagonists. Joumal of Neurochemistry, 16, 1283-1291.

Church, R. M. (1984). Properties of the internal clock. In J. Gibbon \& L. G. Allan (Eds.), Timing and time perception (Annals of the New York Academy of Sciences, Vol. 423, pp. 566-582). New York: New York Academy of Sciences. 
Church, R. M. (1989). Theories of timing behavior. In S. B. Klein \& R. R. Mowrer (Eds.), Contemporary learning theories: Instrumental conditioning theory and the impact of biological constraints on leaming (pp. 41-71). Hillsdale, NJ: Erlbaum.

Church, R. M., MECK, W. H. (1988). Biological basis of the remembered time of reinforcement. In M. L. Commons, R. M. Church, J. R. Stellar, \& A. R. Wagner (Eds.), Quantitative analyses of behavior: Biological determinants of reinforcement (Vol. 7, pp. 103-119). Hillsdale, NJ: Erlbaum.

Church, R. M., Miller, K. D., Meck, W. H., Gibbon, J. (1991). Symmetrical and asymmetrical sources of variance in temporal generalization. Animal Learning \& Behavior, 19, 207-214.

COOPER, J. R., Pincus, J. H. (1967). The role of thiamine in nerve conduction. In G. E. W. Wolstenholme \& M. O'Connor (Eds.), Thiamine deficiency: Biochemical lesions and their clinical significance (Vol. 28, pp. 112-134). Boston: Little, Brown \& Co.

DEws, P. B. (1970). The theory of fixed-interval responding. In W. N. Schoenfield (Ed.), The theory of reinforcement schedules (pp. 43-61). New York: Appleton-Century-Crofts.

Eder, L., HiRT, L., \& Dunant, Y. (1976). Possible involvement of thiamine in acetylcholine release. Nature, 264, 186-188.

Gibbon, J., \& СнuRch, R. M. (1990). Representation of time. Cognition, 37, 23-54.

Gibbon, J., Church, R. M., \& Meck, W. H. (1984). Scalar timing in memory. In J. Gibbon \& L. Allan (Eds.), Timing and time perception (Annals of the New York Academy of Sciences, Vol. 423, pp. 52-77). New York: New York Academy of Sciences.

GUBLER, C. J. (1961). Studies on the physiological functions of thiamine. 1 . The effects of thiamine deficiency and thiamine antagonists on the oxidation of $\alpha$-keto acids by rat tissues. Jourmal of Biological Chemistry, 236, 3112-3120.

HASHIMOTO, C. P. (1981). Effects of repeated administration of pyrithiamine and oxythiamine on timing behavior in rats. Folia Pharmacologica Japonica, 78, 521-528

Heinrich, C. P., Stadler, H., \& Weiser, H. (1973). The effect of thiamine deficiency on the acetylcoenzyme and acetylcholine levels in the rat brain. Journal of Neurochemistry, 21, 1273-1281.

Mair, R. G., Anderson, C. D., Langlais, P. J., \& MCEntee, W. J. (1985). Thiamine deficiency depletes cortical norepinephrine and impairs learning processes in the rat. Brain Research, 360, 273-284

Mair, R. G., Anderson, C. D., Langlais, P. J., McEntee, W. J. (1988). Behavioral impairments, brain lesions and monoaminergic activity in the rat following recovery from a bout of thiamine deficiency. Behavioural Brain Research, 27, 223-239.

MaIR, R. G., \& McEnTEE, W. J. (1983). Korsakoff's psychosis: Noradrenergic systems and cognitive impairment. Behavioural Brain Research, 9, 1-32.

ManN, P. J. G., Quastel, J. H. (1940). Vitamin B1 and acetylcholine formation in isolated brain. Nature, 145, 856-857.

McEntee, W. J., Mair, R. G., \& Langlais, P. J. (1984). Neurochemical pathology in Korsakoff's psychosis: Implications for other cognitive disorders. Neurology, 34, 648-652.

MECK, W. H. (1983). Selective adjustment of the speed of internal clock and memory storage processes. Joumal of Experimental Psychology: Animal Behavior Processes, 9, 171-201.

MECK, W. H. (1987). Vasopressin metabolite neuropeptide facilitates simultaneous temporal processing. Behavioural Brain Research, 23 147-157.

Meck, W. H. (in press). Microinjection of an arginine vasopressin metabolite into the nucleus basalis magnocellularis produces a negativelysigned multiplicative transformation of the content of temporal memory. European Journal of Pharmacology.

MeCK, W. H., СHURCH, R. M. (1987a). Cholinergic modulation of the content of temporal memory. Behavioral Neuroscience, 101 457-464.

MecK, W. H., \& CHURCH, R. M. (1987b). Nutrients that modify the speed of internal clock and memory storage processes. Behavioral Neuroscience, 101, 465-475.

Meck, W. H., ChurCh, R. M., Olton, D. S. (1984). Hippocampus, time, and memory. Behavioral Neuroscience, 98, 3-22.

Meck, W. H., Church, R. M., \& Wenk, G. L. (1986). Arginine vasopressin inoculates against age-related increases in sodiumdependent high affinity choline uptake and discrepancies in the content of temporal memory. European Journal of Pharmacology, 130, 327-331.

Meck, W. H., Church, R. M., \& Wenk, G. L. (in press). Temporal memory in mature and aged rats is sensitive to choline acetyltransferase inhibition. Neurobiology of Aging.

Meck, W. H., ChURCh, R. M., Wenk, G. L., \& Olton, D. S. (1987) Nucleus basalis magnocellularis and medial septal area lesions differentially impair temporal memory. Joumal of Neuroscience, 7, 3505-3511.

Parker, W. D. HaAs, R., Stumpf, D. A., Parks, J., Eguren, L. A \& JACKSON, C. (1984). Brain mitochondrial metabolism in experimental thiamine deficiency. Neurology, 34, 1477-1481.

REYNOLDS, S. F., \& BLASS, J. P. (1975). Normal levels of acetylcoenzyme $A$ and of acetylcholine in the brains of thiamine-deficient rats. Journal of Neurochemistry, 24, 185-186.

Rindi, G., Patrini, C., Comincioli, V., \& Reggiani, C. (1980). Thiamine content and turnover rates of some rat nervous regions, using labeled thiamine as a tracer. Brain Research, 181, 369-380.

RINDI, G., \& PERRI, V. (1961). Uptake of pyrithiamine by tissue of rats. Biochemical Journal, 80, 214.

ROBERTS, S. (1981). Isolation of an internal clock. Joumal of Experimental Psychology: Animal Behavior Processes, 7, 242-268.

Sacchi, O., Ladinsky, H., Prigioni, I., Consolo, S., Peri, G., \& PERR, V. (1978). Acetylcholine turnover in the thiamine-depleted superior cervical ganglion of the rat. Brain Research, 151, 609-614.

Troncoso, J. C., Johnson, M. V., Hess, K. M., Griffin, J. W., \& PRiCE, D. L. (1981). Model of Wernicke's encephalopathy. Archives of Neurology, 38, 350-354.

VorheEs, C. V., SChmidT, D. E., BARRett, R. J., \& SChenker, S. (1977). Effects of thiamine deficiency on acetylcholine levels and utilization in vivo in rat brain. Journal of Nutrition, 107, 1902-1908.

(Manuscript received February 20, 1991; revision accepted for publication September 19, 1991.) 